YCTP-P-04-00

\title{
Low Energy Theory for 2 flavors at High Density QCD
}

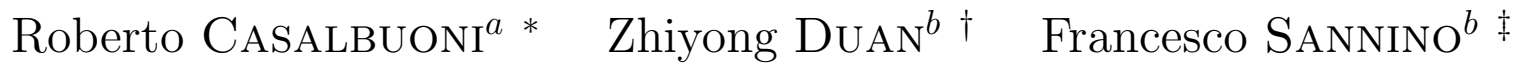 \\ ${ }^{a}$ Dipartimento Di Fisica, Univ. di Firenze and I.N.F.N Sezione di Firenze, \\ I-50125, Italia. \\ ${ }^{b}$ Department of Physics, Yale Univ., New Haven, CT 06520-8120, USA.
}

\begin{abstract}
We construct the effective Lagrangian describing the low energy excitations for Quantum Chromodynamics with two flavors at high density. The non-linear realization framework is employed to properly construct the low energy effective theory. The light degrees of freedom, as required by 't Hooft anomaly conditions, contain massless fermions which we properly include in the effective Lagrangian. We also provide a discussion of the linearly realized Lagrangian.
\end{abstract}

*Electronic address: Roberto.Casalbuoni@fi.infn.it

$\dagger$ Electronic address: zhiyong.duan@yale.edu

$\ddagger$ Electronic address: francesco.sannino@yale.edu 


\section{INTRODUCTION}

Recently quark matter at very high density has attracted a great flurry of interest [1 15 . In this region, quark matter is expected to behave as a color superconductor [1,2]. Possible phenomenological applications are associated with the description of neutron star interiors, neutron star collisions and the physics near the core of collapsing stars. A better understanding of highly squeezed nuclear matter might also shed some light on nuclear matter at low density, i.e. densities close to ordinary nuclear matter where some models already exist. For example, in Ref. [6] a rather complete soliton model at low density is constructed containing vector-bosons, along with the Goldstone bosons.

In a superconductive phase, the color symmetry is spontaneously broken and a hierarchy of scales, for given chemical potential, is generated. Indicating with $g$, the underlying coupling constant, the relevant scales are: the chemical potential $\mu$ itself, the dynamically generated gluon mass $m_{\text {gluon }} \sim g \mu$ and the gap parameter $\Delta \sim \frac{\mu}{g^{5}} e^{-\frac{\alpha}{g}}$ with $\alpha$ a calculable constant. Since for high $\mu$ the coupling constant $g$ (evaluated at the fixed scale $\mu$ ) is $\ll 1$, we have:

$$
\Delta \ll m_{\text {gluon }} \ll \mu
$$

Massless excitations dominate physical processes at very low energy with respect to the energy gap $(\Delta)$. Their spectrum is intimately related to the underlying global symmetries and the way they are realized at low energies. Indeed when the dynamics is such that a continuous global symmetry is spontaneously broken, a Goldstone boson appears in order to compensate for the breaking. Massless excitations obey low energy theorems governing their interactions which can be usefully encoded in effective Lagrangians. A well known example, in the region of cold and non-dense QCD, is the effective Lagrangian for pions and kaons. These Lagrangians are seen to describe well the QCD low energy phenomenology [7].

Another set of relevant constraints is provided by quantum anomalies. At zero density and temperature, 't Hooft [8] argued that the underlying continuous global anomalies have to be matched in a given low energy phase by a set of massless fermions associated with the intact global symmetries and a set of massless Goldstone bosons associated with the broken ones. The low energy fermions (composite or elementary) contribute via triangle diagrams while for the Goldstones a Wess-Zumino term should be added to correctly implement the associated global anomalies.

In [9], the 't Hooft constraints were seen to hold for QCD at finite density. In particular, it was shown, by reviewing the dynamically favored phases for $N_{f}=2,3$ at high density, that 
the low energy spectrum displayes the correct quantum numbers to saturate the 't Hooft global anomalies. It was also observed that QCD at finite density can be envisioned, from a global symmetry and anomaly point of view, as a chiral gauge theory [10,11] for which at least part of the matter field content is in complex representations of the gauge group. Indeed an important distinction from zero density, vector-like theories is that these theories, when strongly coupled, can exist in the Higgs phase by dynamically breaking their own gauge symmetries [10,11]. This is also the striking feature of the superconductive phase allowed for QCD at high density. In fact at finite density, vector-like symmetries are no longer protected against spontaneous breaking by the Vafa-Witten theorem [12].

In this paper we build the effective theory describing the low energy excitations for Quantum Chromodynamics with two flavors at high density. The non linear realization framework [13] is used to properly construct the low energy theory. The light degrees of freedom, as required by 't Hooft anomaly conditions, contains massless fermions which we properly include in the effective Lagrangian. We prove that the non linearly realized low energy effective theory, in general, contains at the lowest order in a derivative expansion for the Goldstones (i.e. two derivative) two independent terms which are responsible for distinct contributions to the gluon masses [14] when gauging the color degrees of freedom.

We finally provide a discussion of the linearly realized Lagrangian. In this framework we see, as in reference [14,15, that the linearly realized effective Lagrangian at the dimension four level actually predicts the squared gluon mass ratio between the eighth gluon and the other massive ones to be $4 / 3$. This result does not agree with the underlying calculations [14]. In order to resolve the issue, in Ref. [14,15] it was pointed out that one can include in the linear Lagrangian another two derivative term possessing mass dimension 6 . This result shows the failure of the standard naive dimensional counting arguments for suppressing higher dimensional terms for the effective linearly realized effective Lagrangian. Interestingly, in the non linear realization case all the terms needed to correctly describe the gluon masses arises at the same derivative order. This fact suggests that non linear realization description leads to a consistent counting scheme.

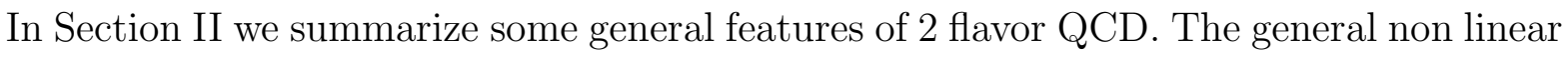
formalism for the spontaneous breaking of color is presented in Section 111 . We implement the breaking of the baryon number in Section IV. Then, in medium, fermions are introduced in Section $\square$ together with the summary of the low energy theory and its extension to the broken Lorentz group case. A linearly realized Lagrangian is explored in Section VI. In Section VII we conclude. 


\section{GENERAL FEATURES OF 2 FLAVOR QCD AT HIGH DENSITY}

The underlying gauge group is $S U(3)$ while the quantum flavor group is

$$
S U_{L}(2) \times S U_{R}(2) \times U_{V}(1)
$$

and the classical $U_{A}(1)$ symmetry is destroyed at the quantum level by the Adler-BellJackiw anomaly. We indicate with $q_{L c, i ; \alpha}$ the two component left spinor where $\alpha=1,2$ is

the spin index, $c=1,2,3$ is the color index while $i=1,2$ represents the flavor. $q_{R_{c, i}}^{\dot{\alpha}}$ is the two component right spinor. We summarize the transformation properties in the following table:

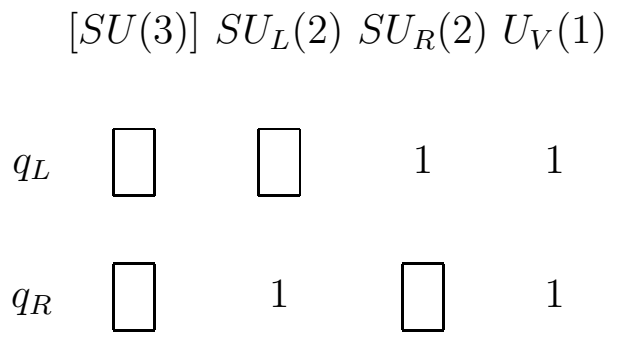

where $S U(3)$ is the gauge group, indicated by square bracket. The theory is subject to the following global anomalies:

$$
S U_{L / R}(2)^{2} U_{V}(1) \propto \pm 3
$$

where we have chosen a convention for the flavor generators in which the quadratic anomaly factor is 1 .

At zero density we have two possible phases compatible with the anomaly conditions. One is the ordinary Goldstone phase associated with the spontaneous breaking of the underlying global symmetry to $S U_{V}(2) \times U_{V}(1)$. The other is the Wigner-Weyl phase where, assuming confinement, the global symmetry at low energy is intact and the needed massless spectrum consists of massless baryons. The Goldstone phase, associated with a non vanishing $\bar{q}_{L} q_{R}$ condensate, is the one observed in nature. This fact supports a new idea presented in Ref. [11]. There it is suggested that, for an asymptotically free theory, among multiple infrared phases allowed by 't Hooft anomaly conditions, the one which minimizes the entropy at the approach to freeze-out is preferred.

What happens when we squeeze nuclear matter? At very low baryon density compared to a fixed intrinsic scale of the theory $\Lambda$, it is reasonable to expect that the Goldstone phase persists. On the other hand at very large densities, it is seen, via dynamical calculations [1,2], that the ordinary Goldstone phase is no longer favored compared with a superconductive one associated with the following type of quark condensates: 


$$
\epsilon^{\alpha \beta} \epsilon^{a b c} \epsilon^{i j}<q_{L b, i ; \alpha} q_{L c, j ; \beta}>, \quad \epsilon_{\dot{\alpha} \dot{\beta}} \epsilon^{a b c} \epsilon^{i j}<q_{R b, i}^{\dot{\alpha}} q_{R c, j}^{\dot{\beta}}>.
$$

We can now introduce two scalar fields which play the role of order parameters:

$$
L^{\dagger^{a}} \sim \epsilon^{a b c} \epsilon^{i j} q_{L b, i}^{\alpha} q_{L c, j ; \alpha}, \quad R^{\dagger a} \sim-\epsilon^{a b c} \epsilon^{i j} q_{R b, i ; \dot{\alpha}} q_{R c, j}^{\dot{\alpha}} .
$$

Under a parity transformation

$$
L_{a} \leftrightarrow R_{a}
$$

If parity is not broken spontaneously, we have

$$
\left\langle L_{a}\right\rangle=\left\langle R_{a}\right\rangle=v \delta_{a}^{3},
$$

where we chose the condensate to be in the 3rd direction of color. This condensate is not allowed at zero density by the Vafa-Witten theorem. The order parameters are singlets under the $S U_{L}(2) \times S U_{R}(2)$ flavor transformations while possessing baryon charge 2 .

The vev breaks the gauge symmetry while leaving intact the following group:

$$
[S U(2)] \times S U_{L}(2) \times S U_{R}(2) \times \widetilde{U}_{V}(1),
$$

where $[S U(2)]$ is the unbroken part of the gauge group. The $\widetilde{U}_{V}(1)$ generator is the following linear combination of the previous $U_{V}(1)$ generator $Q=\operatorname{diag}(1,1,1)$ and the broken diagonal generator of the $S U(3)$ gauge group $T^{8}=\frac{1}{2 \sqrt{3}} \operatorname{diag}(1,1,-2)$

$$
\widetilde{S}=\frac{1}{3 \sqrt{2}}\left[Q-2 \sqrt{3} T^{8}\right] .
$$

The $\widetilde{S}$ charge of the quarks with color 1 and 2 is zero.

The superconductive phase for $N_{f}=2$ possesses the same global symmetry group of the confined Wigner-Weyl phase. This remarkable feature when considering chiral gauge theories at zero density (where the superconductive phase is now a Higgs phase) is referred as complementarity. This idea was introduced in Ref. [16] where it was conjectured that any Higgs phase can be described in terms of confined degrees of freedom and vice versa.

It is convenient to introduce the fields:

$$
V_{a}=\frac{L_{a}+R_{a}}{\sqrt{2}}, \quad A=\frac{L_{a}-R_{a}}{\sqrt{2}} .
$$

On the vacuum, via (2.7), we have:

$$
\left\langle V_{a}\right\rangle=\sqrt{2} v \delta_{a}^{3} \quad\left\langle A_{a}\right\rangle=0 .
$$


The massless excitations are associated with the fluctuations around the vacuum expectation value for $V_{a}$ while the fields described by $A_{a}$ are massive. In Ref. [17] it is argued, based on a dynamical calculation, that at very high density the $A_{a}$ fields might be very light and possibly relevant for the low energy phenomenology. However we will focus mainly on the truly massless excitations since low energy theorems are valid only for these fields. Low energy theorems are, in general, efficiently encoded in a non linear realization framework which we will soon construct.

\section{LOW ENERGY EFFECTIVE THEORY WITHOUT BARYON NUMBER}

In order to write an effective Lagrangian for 2 flavor $\mathrm{QCD}$, one could, at least in principle, proceed in different ways. For example one could add a mass term for the strange quark in the 3 flavor Lagrangian. However, since the theory is not supersymmetric, an exact decoupling procedure is not known at effective Lagrangian level. A general way, which we will explore here, to directly construct the 2 flavor effective Lagrangian makes use of the non linear realization methods [13]. The latter have been already successfully employed for the 3 flavor case at high density in [19].

Following reference [19], we now construct the non linearly realized effective Lagrangian containing the diquark degrees of freedom coupled to gluons.

We postpone the breaking of the baryon number and its consequences to the next section. The group of transformations is $G=S U(3)$, while the stability group leaving the vacuum invariant is the proper subgroup $H=S U(2) \subset G$. The color generators $T^{m}$ of $S U(3)$, with $m=1, \ldots, 8$, obey the normalization condition $\operatorname{Tr}\left[T^{m} T^{n}\right]=\frac{1}{2} \delta^{m n}$. We divide the generators $\{T\}$ into two classes, calling the generators of $H\left\{S^{a}=T^{a}\right\}$, with $a=1, \ldots, 3$ and the broken generators $\left\{X^{i}=T^{i+3}\right\}$ with $i=1, \ldots, 5$. It is worth noticing that the quotient space $G / H$, in this case, is not a symmetric space. By symmetric space we mean that, if $X$ and $S$ represent arbitrary linear combinations of the broken and unbroken generators, their commutators should satisfy the restriction:

$$
[X, X]=i S
$$

It is easy to verify that the previous condition is not obeyed. Clearly, our generators should always satisfy the trivial conditions

\footnotetext{
$\S$ An attempt to generalize to QCD the Seiberg decoupling procedure at the effective Lagrangian level is provided in [18].
} 


$$
[S, S]=i S, \quad[X, S]=i X
$$

expressing the fact that the Lie algebra of $H$ closes and that the $\{X\}$ form a representation of $H$.

The coset space $G / H$ is parameterized by the group elements

$$
\mathcal{V}=\exp \left(i \xi^{i} X^{i}\right)
$$

with $\xi^{i}=\Pi^{i} / v$ describing the Goldstone fields and are coordinates of the space $G / H . \mathcal{V}$ transforms non linearly under a color transformation, i.e.:

$$
\mathcal{V}(\xi) \rightarrow g \mathcal{V}(\xi) h^{\dagger}(\xi, g)
$$

where $g \in G$ and $h \in H$. It is convenient to define the hermitian (algebra valued) MaurerCartan one-form

$$
\omega_{\mu}=i \mathcal{V}^{\dagger} D_{\mu} \mathcal{V},
$$

with $D_{\mu}$ the covariant derivative with respect to $G=S U(3)$

$$
D_{\mu} \mathcal{V}=\left(\partial_{\mu}-i G_{\mu}\right) \mathcal{V}
$$

and $G_{\mu}^{m} T^{m}$ the gluon fields. Since $\mathcal{V}$ transforms with respect to $G$ as in Eq. (3.4) it follows that

$$
\omega_{\mu} \rightarrow h(\xi, g) \omega_{\mu} h^{\dagger}(\xi, g)+h(\xi, g) \partial_{\mu} h^{\dagger}(\xi, g) .
$$

We decompose $\omega_{\mu}$ into the part parallel to $H$

$$
\omega_{\mu}^{\|}=2 S^{a} \operatorname{Tr}\left[S^{a} \omega_{\mu}\right], \quad \in \text { Lie } H,
$$

and into the perpendicular part

$$
\omega_{\mu}^{\perp}=2 X^{i} \operatorname{Tr}\left[X^{i} \omega_{\mu}\right], \quad \in \text { Lie } G-\text { Lie } H
$$

Summation over repeated indices is assumed.

This yields the following transformation properties:

$$
\omega_{\mu}^{\|} \rightarrow h(\xi, g) \omega_{\mu}^{\|} h^{\dagger}(\xi, g)+h(\xi, g) \partial_{\mu} h^{\dagger}(\xi, g),
$$

and

$$
\omega_{\mu}^{\perp} \rightarrow h(\xi, g) \omega_{\mu}^{\perp} h^{\dagger}(\xi, g) .
$$


Then, it turns out that the most general invariant of second order in the derivatives is

$$
L=v^{2} \operatorname{Tr}\left[\omega_{\mu}^{\perp} \omega^{\mu \perp}\right]=-2 v^{2} \operatorname{Tr}\left[X^{i} \mathcal{V}^{\dagger} D_{\mu} \mathcal{V}\right] \operatorname{Tr}\left[X^{i} \mathcal{V}^{\dagger} D^{\mu} \mathcal{V}\right]
$$

We can immediately see, by adopting the unitary gauge (corresponding to $\xi \rightarrow 0$ ), that the previous term provides a mass term for the gluons associated with the 5 coset generators, whereas the gluons $G^{1,2,3}$ remain massless. Indeed the mass term reads:

$$
L=2 v^{2} \operatorname{Tr}\left[X^{i} G_{\mu}\right] \operatorname{Tr}\left[X^{i} G^{\mu}\right]=\frac{v^{2}}{2} G_{\mu}^{i} G^{i \mu}
$$

The fact that we find the same mass for all of the gluons of the broken subgroup is not surprising, since we have treated them equally. In the next section we address the problem of the baryon number and show how, in the non linear framework, this naturally leads to a distinct mass for the eighth gluon.

The massless gluons of the subgroup $H$ confine, leaving, as we shall see, only some of the quarks as massless excitations at low energies.

\section{THE ABELIAN GROUP $U_{V}(1)$}

The previous discussion is not yet complete, since we have omitted the breaking of the baryon number. Indeed the group of transformations should be $G=S U(3) \times U_{V}(1)$, while the unbroken subgroup is $H=S U(2) \times \widetilde{U}_{V}(1)$.

The non linear transformations are now:

$$
\begin{gathered}
\mathcal{V}(\xi) \rightarrow u_{V} g \mathcal{V}(\xi) h^{\dagger}(\xi, g, u) h_{\widetilde{V}}^{\dagger}(\xi, g, u) \\
u_{V} \in U_{V}(1), \quad g \in S U(3), \quad h(\xi, g, u) \in S U(2), \quad h_{\widetilde{V}}(\xi, g, u) \in \widetilde{U}_{V}(1) .
\end{gathered}
$$

The $U_{V}(1)$ charge is $Q=\operatorname{diag}(1,1,1)$. The generator, for $\widetilde{U}_{V}(1)$, leaving the vev invariant is

$$
\widetilde{S}=\frac{1}{3 \sqrt{2}}\left[Q-2 \sqrt{3} T^{8}\right]=\operatorname{diag}(0,0, \sqrt{2} / 2)
$$

where we have chosen to normalize it according to $\operatorname{Tr}\left[\widetilde{S}^{2}\right]=1 / 2$. The coset space $G / H$ is parameterized, as before, by the group elements

$$
\mathcal{V}=\exp \left(i \xi^{i} X^{i}\right)
$$

but now we identify the Goldstone bosons as: 


$$
\begin{aligned}
\xi^{i} & =\frac{\Pi^{i}}{v} \quad i=1,2,3,4, \\
\xi^{5} & =\frac{\Pi^{5}}{\widetilde{v}} .
\end{aligned}
$$

We still have 5 generators $\left\{X^{i}\right\}$ which belong to the coset space $G / H$, but one of them needs to be modified. While $X^{1,2,3,4}$ are still identified, respectively, with $T^{4,5,6,7}$, the coset generator which replaces $X^{5}=T^{8}$ of the previous section is now

$$
X^{5}=\frac{1}{3}\left[Q+\sqrt{3} T^{8}\right]=\operatorname{diag}\left(\frac{1}{2}, \frac{1}{2}, 0\right) .
$$

We used the orthogonality condition

$$
\operatorname{Tr}[X S]=0
$$

to construct $X^{5}$ with $\operatorname{Tr}\left[X^{5} X^{5}\right]=\frac{1}{2}$. Notice that $X^{5}$ is no longer traceless (i.e. $\operatorname{Tr}\left[X^{5}\right]=1$ ).

It is amusing to note that $\mathcal{V}$ transforms on the left as an ordinary quark with respect to color and $U_{V}(1)$ transformations. This will turn to be important when adding the fermions.

As for ordinary QCD, we can construct a field transforming linearly by using only non linearly realized fields via:

$$
V_{a}=\frac{v}{\sqrt{2}} \epsilon_{a b c} \mathcal{V}_{i}^{b} \mathcal{V}_{j}^{c} \epsilon^{i j 3}
$$

To prove that the previous expression transforms linearly we recall that the $H$ subgroup acts on the right of $\mathcal{V}$. So under a general non linear transformation we have:

$$
\epsilon_{a b c} \mathcal{V}_{i}^{b} \mathcal{V}_{j}^{c} \epsilon^{i j 3} \rightarrow u_{V}^{2} \epsilon_{a b c} g_{d}^{b} g_{e}^{c} \mathcal{V}_{k}^{d} \mathcal{V}_{l}^{e}{h^{\dagger}}_{i}^{k} h^{\dagger}{ }_{j} h_{\widetilde{V}}^{\dagger} \epsilon^{i j 3} \rightarrow u_{V}^{2} \epsilon_{a b c} g_{d}^{b} g_{e}^{c} \mathcal{V}_{k}^{d} \mathcal{V}_{l}^{e} \epsilon^{k l 3}
$$

Indeed due to Eq. (4.3) we have that $h_{\widetilde{V}}$ acting on the color indices 1 and 2 is equivalent to the identity. We also have

$$
h_{i}^{\dagger}{ }_{i}^{\dagger}{ }_{j}^{\dagger} \epsilon^{i j 3}=\epsilon^{k l 3}
$$

since $h$ is an $S U(2)$ matrix and we have conveniently chosen the indices. Therefore, $V_{a}$ transforms linearly. Using the relation:

$$
\operatorname{det} \mathcal{V} \equiv \frac{1}{3 !} \epsilon_{a b c} \epsilon^{k i j} \mathcal{V}_{k}^{a} \mathcal{V}_{i}^{b} \mathcal{V}_{j}^{c}=\exp \left[i \frac{\Pi^{5}}{\widetilde{v}} \frac{\operatorname{Tr}[Q]}{3}\right]=\exp \left[i \frac{\Pi^{5}}{\widetilde{v}}\right]
$$

we can rewrite Eq. (4.9) in the following form:

$$
V_{a}=\sqrt{2} v e^{i \frac{\Pi^{5}}{v}} \mathcal{V}_{a}^{-1^{3}}
$$


The latter expression corresponds to the polar decomposition of the vector $V_{a}$ with the massive scalar field frozen to its vev value. Since the field $\Pi^{5}$ is associated with the $X^{5}$ generator, which is a linear combination of an abelian and of a non abelian $T^{8}$ generator, $\operatorname{det} \mathcal{V}$ does not parameterize an independent field.

Expanding $\mathcal{V}$ up to first order in the Goldstones leads to:

$$
V_{a}=\sqrt{2} v\left[1+i \xi^{5}\left(\frac{2}{3} Q-\frac{1}{\sqrt{3}} T^{8}\right)-i \sum_{i=1}^{4} \xi^{i} X^{i}\right]_{a}^{c} \delta_{c}^{3}+v \mathcal{O}\left(\xi^{2}\right) .
$$

This new field explicitly describes the vev properties. First of all, when considering the limit $\xi \rightarrow 0$ we recover the correct vacuum, i.e.

$$
V_{a}=\sqrt{2} v \delta_{a}^{3} .
$$

Furthermore, as expected, $V_{a}$ transforms under the underlying gauge transformations as a diquark.

Equation (4.9) mimics what happens in ordinary QCD. There the vev transformations are encoded in the linearly transforming matrix $U \rightarrow g_{L} U g_{R}^{\dagger}$ while the non linearly transforming field is simply $\sqrt{U} \rightarrow g_{L} \sqrt{U} k^{\dagger}\left(\sqrt{U}, g_{L}, g_{R}\right)=k\left(\sqrt{U}, g_{L}, g_{R}\right) \sqrt{U} g_{R}^{\dagger}$, with $k\left(\sqrt{U}, g_{L}, g_{R}\right) \in$ $S U_{V}(3)$.

If we insist in gauging the color transformations the general discussion in the previous section remains unchanged. The substantial difference is that now the most generic two derivative kinetic Lagrangian

$$
L=v^{2} a_{1} \operatorname{Tr}\left[\omega_{\mu}^{\perp} \omega^{\mu \perp}\right]+v^{2} a_{2} \operatorname{Tr}\left[\omega_{\mu}^{\perp}\right] \operatorname{Tr}\left[\omega^{\mu \perp}\right],
$$

acquires a new term. The presence of a double trace term is due to the absence of the traceless condition for the broken generator $X^{5}$.

The kinetic term for the Goldstones is:

$$
L_{k i n}=\frac{a_{1}}{2} \sum_{i=1}^{4} \partial_{\mu} \Pi^{i} \partial^{\mu} \Pi^{i}+\frac{v^{2}}{\widetilde{v}^{2}}\left(\frac{a_{1}+2 a_{2}}{2}\right) \partial_{\mu} \Pi^{5} \partial^{\mu} \Pi^{5} .
$$

Normalizing the kinetic term we have

$$
a_{1}=1, \quad a_{2}=\frac{1}{2}\left[\frac{\widetilde{v}^{2}}{v^{2}}-a_{1}\right] .
$$

The square gluon mass ratio, in the unitary gauge (i.e. $\xi \rightarrow 0$ ), between the eighth gluon and any other massive one now reads:

$$
\frac{m_{8}^{2}}{m_{i}^{2}}=\frac{1}{3}\left[1+2 \frac{a_{2}}{a_{1}}\right]=\frac{1}{3} \frac{\widetilde{v}^{2}}{v^{2}}
$$


with $i=4,5,6$ or 7 and in the last step we use Eq. (4.18). Using perturbation theory at one loop [14] $a_{2} \approx 1 / 2$ (i.e. $\widetilde{v} \approx \sqrt{2} v$ ). Also, as we shall see, in the linearly realized lagrangian one can write [15] two two-derivative terms in the effective Lagrangian. However one of them appears in the Lagrangian as a higher order in mass dimension, leading to a not straightforward systematic expansion.

\section{IN MEDIUM FERMIONS}

Now we turn our attention to the Fermi fields. First of all, let us introduce the following field:

$$
\widetilde{\psi}=\mathcal{V}^{\dagger} \psi
$$

which under the action of the group $G=S U(3) \times U_{V}(1)$ transforms as

$$
\widetilde{\psi} \rightarrow h_{\widetilde{V}}(\xi, g, u) h(\xi, g, u) \widetilde{\psi} .
$$

This construction allows us to easily switch to the non linear representations and it is often encountered in the Heavy Quark Effective formalism [20].

The fermion field in (5.1) can be thought as a, in medium, quark surrounded by a Goldstone cloud. Remarkably, as also required by 't Hooft anomaly conditions [9], the newly

defined (in medium) fermion possesses the unbroken $\widetilde{U}_{V}(1)$ charge. The cloud (effectively included in $\mathcal{V}$ ) correctly screens the baryonic charge.

In the new variables, one can construct two independent invariant terms

$$
L_{1}=\overline{\widetilde{\psi}} i \gamma^{\mu}\left(\partial_{\mu}-i \omega_{\mu}^{\|}\right) \widetilde{\psi}, \quad L_{2}=\overline{\widetilde{\psi}} \gamma^{\mu} \omega_{\mu}^{\perp} \widetilde{\psi} .
$$

One can easily verify that the tree level lagrangian term for the quarks

$$
L_{\text {Tree }}=\bar{\psi} \gamma^{\mu}\left(\partial_{\mu}-i G_{\mu}\right) \psi,
$$

corresponds to the linear combination of

$$
L_{\text {Tree }}=L_{1}+L_{2} .
$$

Another invariant that we can add to the effective Lagrangian corresponds to the following Majorana mass term:

$$
L_{m}=m_{M} \bar{\psi}^{C}{ }_{i} \gamma^{5}\left(i T^{2}\right) \tilde{\psi}_{j} \epsilon^{i j}+\text { h.c. }
$$


with $\tilde{\psi}^{C}=i \gamma^{2} \tilde{\psi}^{*}$, where $i, j=1,2$ are flavor indices. The invariance under a $h(\xi, g, u) \in$ $S U(2)$ transformation is insured by the relation

$$
\left(S^{a}\right)^{T} T^{2}=-T^{2} S^{a}
$$

where

$$
T^{2}=S^{2}=\frac{1}{2}\left(\begin{array}{ll}
\sigma^{2} & 0 \\
0 & 0
\end{array}\right)
$$

The invariance under a $\tilde{U}_{V}(1)$ transformation is due to the fact that its generator $\tilde{S}=$ $\operatorname{diag}\left(0,0, \frac{\sqrt{2}}{2}\right)$ is such that $(\tilde{S})^{T} T^{2}=-T^{2} \tilde{S}=0$.

We stress that this Lagrangian term respects the underlying color transformations as well as the global transformations $S U_{L}(2) \times S U_{R}(2) \times U_{V}(1)$. The breaking of the underlying symmetries manifests itself only when we evaluate this Lagrangian on the vev (i.e. $\xi \rightarrow 0$ ). To see this one can rewrite the previous terms as an explicit function of $\mathcal{V}$. Parity is also enforced. This term does not produce a mass term for the quarks with color index 3 while yielding a Majorana mass term for fermions with color index 1 and 2 .

The latter Lagrangian term is similar to the meson-fermion interaction we can write in standard QCD (of the type $\bar{q} U q$ which yields the constituent Dirac quark masses when evaluated on the vev).

At this point, for reader's convenience, we summarize the total non linearly realized effective Lagrangian describing in medium fermions, gluons and their self interactions, up to two derivatives,

$$
\begin{aligned}
\mathcal{L}= & \quad v^{2} a_{1} \operatorname{Tr}\left[\omega_{\mu}^{\perp} \omega^{\mu \perp}\right]+v^{2} a_{2} \operatorname{Tr}\left[\omega_{\mu}^{\perp}\right] \operatorname{Tr}\left[\omega^{\mu \perp}\right] \\
+ & b_{1} \overline{\widetilde{\psi}} i \gamma^{\mu}\left(\partial_{\mu}-i \omega_{\mu}^{\|}\right) \tilde{\psi}+b_{2} \overline{\widetilde{\psi}} \gamma^{\mu} \omega_{\mu}^{\perp} \widetilde{\psi} \\
+ & m_{M} \overline{\tilde{\psi}^{C}} \gamma^{5}\left(i T^{2}\right) \tilde{\psi}+\text { h.c. }
\end{aligned}
$$

Here $a_{1}, a_{2}, b_{1}$ and $b_{2}$ are real coefficients while $m_{M}$ is complex and we omit flavor indices. At the tree level in the underlying theory we have $b_{1}=b_{2}=1$. The massless degrees of freedom are the in medium fermions $\tilde{\psi}_{a=3, i}$ which possess the correct quantum numbers prescribed by 't Hooft anomaly conditions [9]. As already mentioned, the $S U(2)$ color subgroup remains unbroken and the associated 3 gluons are expected to confine again. To the previous general effective Lagrangian we should also add the gluon kinetic term.

In writing the effective low energy theory we have not yet considered the breaking of Lorentz invariance at finite density. Following Ref. [19] we impose invariance only under the 
$O(3)$ subgroup of the Lorentz transformations. This amounts to have different coefficients for the temporal and spatial indices of the Lagrangian which now becomes:

$$
\begin{aligned}
\mathcal{L}= & v^{2} a_{1} \operatorname{Tr}\left[\omega_{0}^{\perp} \omega_{0}^{\perp}-\alpha_{1} \vec{\omega}^{\perp} \vec{\omega}^{\perp}\right]+v^{2} a_{2}\left[\operatorname{Tr}\left[\omega_{0}^{\perp}\right] \operatorname{Tr}\left[\omega_{0}^{\perp}\right]-\alpha_{2} \operatorname{Tr}\left[\vec{\omega}^{\perp}\right] \operatorname{Tr}\left[\vec{\omega}^{\perp}\right]\right] \\
+ & b_{1} \overline{\widetilde{\psi}} i\left[\gamma^{0}\left(\partial_{0}-i \omega_{0}^{\|}\right)+\beta_{1} \vec{\gamma} \cdot\left(\vec{\nabla}-i \vec{\omega}^{\|}\right)\right] \widetilde{\psi}+b_{2} \bar{\psi}\left[\gamma^{0} \omega_{0}^{\perp}+\beta_{2} \vec{\gamma} \cdot \vec{\omega}^{\perp}\right] \widetilde{\psi} \\
& +m_{M} \overline{\tilde{\psi}^{C}} \gamma^{5}\left(i T^{2}\right) \tilde{\psi}+\text { h.c. }
\end{aligned}
$$

where the new coefficients $\alpha$ s and $\beta$ s encode the effective breaking of Lorentz invariance. To the previous Lagrangian we can still add the chemical potential type of term $\overline{\widetilde{\psi}} \gamma^{0} \widetilde{\psi}$.

Clearly, in future, it would be valuable to compute the coefficients of the effective Lagrangian in Eq. (5.10) at asymptotically high densities. We notice that, due to the invariance under the global $S U_{L}(2) \times S U_{R}(2)$ symmetry group, no mass term arises for the third colored quarks and that any dynamical calculation (preserving the flavor symmetries) will have to respect this condition. In Ref. [21], using some dynamical calculation, it is argued that this seems to be the case.

\section{LINEAR REALIZATIONS}

For the linearly realized effective Lagrangian we can start directly with the triplet field $V_{a}$. This complex field encodes the five Goldstones plus a massive scalar. The relation with the non linearly realized fields is provided in Eq. (4.9).

While in the non linear realization case the effective Lagrangian is ordered in number of derivatives, usually in the linear case, the terms are ordered according to their increasing mass dimension. The general dimension four effective Lagrangian is:

$$
\mathcal{L}=D^{\mu} V^{\dagger} D_{\mu} V+P(V)
$$

$P(V)$ is a potential term of the general form:

$$
P(V)=-M_{V}^{2} V^{\dagger} V+\lambda_{V}\left[V^{\dagger} V\right]^{2}
$$

with $M_{V}$ and $\lambda_{V}$ real parameters and the negative sign mass term has been chosen to provide a non zero vev for $V$

The color covariant derivative is defined as follows:

$$
D_{\mu} V=\partial_{\mu} V+i V G_{\mu}
$$

and $G_{\mu}=G_{\mu}^{m} T^{m}$ is the gluon field. In constructing the previous lagrangian, we have also assumed parity invariance. The potential leads to a non vanishing vacuum expectation value for $V$ which we choose to align in the color direction 3 , 


$$
\left\langle V_{a}\right\rangle=\sqrt{2} v \delta_{a}^{3}, \quad v=\frac{1}{2} \sqrt{\frac{M_{V}^{2}}{\lambda_{V}}} .
$$

The local $S U(3)$ gauge invariance is then spontaneously broken to the $S U(2)$ local gauge subgroup. 5 of the 6 independent scalars contained in $V_{a}$ are massless. We choose the unitary gauge and absorbe the massless degrees of freedom in the longitudinal components of the 5 massive gauge fields. In this gauge we can write the fluctuations around the vacuum for $V_{a}$ as:

$$
V=\left(\begin{array}{c}
0 \\
0 \\
\sqrt{2} v+\sigma
\end{array}\right)
$$

where the scalar $\sigma$ is a massive real field with $\langle\sigma\rangle=0$. The masses of the 5 gluons are related to the condensate via:

$$
m_{i}=v, \quad m_{8}=\frac{2}{\sqrt{3}} v,
$$

with $i=4,5,6,7$. Clearly no Goldstone boson survives since there is no global symmetry left unbroken. As expected the massless degrees of freedom are the first 3 gluons for the unbroken $S U(2)$ gauge symmetry. However the latter are supposed to confine and hence to generate a new confining scale associated with pure gluon-dynamics with two colors but no flavors.

We immediately notice, as in reference [14,15, that the linearly realized effective Lagrangian at the dimension four level predicts the squared gluon mass ratio between the eighth gluon and the other massive ones to be $4 / 3$. This result does not agree with the underlying calculations [14]. In order to resolve the issue in Ref. [14,15], it was pointed out that one can include in the linear Lagrangian another two derivative term of the form:

$$
\frac{b}{v^{2}}\left[V^{\dagger} D_{\mu} V\right]\left[V^{\dagger} D^{\mu} V\right]
$$

$b$ is a real number. This term has mass dimension 6. At this point we cannot use, in general, the standard naive dimensional counting arguments for suppressing other higher dimensional terms for the effective linearly realized Lagrangian. Interestingly, in the non linear realization case, all the terms needed to correctly describe the gluon masses arises at the same derivative order. This fact suggests that non linear realization description leads to the correct counting scheme. 


\section{CONCLUSIONS}

We constructed the low energy effective theory describing Quantum Chromodynamics with two flavors at high density. In order to correctly implement the low energy theorems, we have used the non linear realization framework. An important difference with respect to the 3 flavor case is the presence, guaranteed by 't Hooft anomaly conditions, of massless fermions. We have hence properly added the, in medium, fermions to the effective Lagrangian. Then we generalized it to the case when Lorentz invariance is broken, by medium effects, to $O(3)$. We also show that there are two independent two derivative terms for the Goldstone bosons (which will eventually become longitudinal components of the color gauge bosons). This is shown to be an effect related to the baryon number violation. We finally investigate the linearly realized effective Lagrangian which, in general, is useful and well defined only when describing a phase transition. Here we see, as also noted in Ref. [14, 15], that the naive (in mass) dimensional counting rule used to naturally order the terms does not hold when confronted with perturbative dynamical calculations [14]. In contrast the non linearly realized theory provided a consistent counting scheme.

\section{Acknowledgments}

It is pleasure for us to thank D.H. Rischke and J. Schechter for interesting discussions and encouragement. The work of Z.D. and F.S. has been partially supported by the US DOE under contract DE-FG-02-92ER-40704.

[1] M. Alford, K. Rajagopal and F. Wilczek, Phys. Lett. B422, 247 (1998).

[2] R. Rapp, T. Schäfer, E. V. Shuryak and M. Velkovsky, Phys. Rev. Lett. 81, 53 (1998).

[3] M. Alford, K. Rajagopal and F. Wilczek, Nucl. Phys. B537, 443 (1999).

[4] For recent reviews see: M. Alford, hep-ph/0003185; S. Hsu, hep-ph/0003140; D. H. Rischke and R. D. Pisarski, nucl-th/0004016.

[5] T. Schäfer and F. Wilczek, Phys. Rev. Lett. 82 , 3956 (1999). D. T. Son, Phys. Rev. D59, 094019 (1999). S. C. Frautschi, Asymptotic freedom and color superconductivity in dense quark 
matter, in: Proceedings of the Workshop on Hadronic Matter at Extreme Energy Density, N. Cabibbo, Editor, Erice, Italy (1978). F. Barrois, Nucl. Phys. B129, 390 (1977). D. Bailin and A. Love, Phys. Rept. 107, 325 (1984). S. Elitzur, Phys. Rev. D12, 3978 (1975). E. Fradkin and S. Shenker, Phys. Rev. D19, 3682 (1979). T. Schäfer and F. Wilczek, Phys. Lett. B450, 325 (1999). N. Evans, S. Hsu and M. Schwetz, Nucl. Phys. B551, 275 (1999); Phys. Lett. B449, 281 (1999). M. Alford, J. Berges and K. Rajagopal, Nucl. Phys. B558, 219 (1999). S. Weinberg, Nucl. Phys. B413, 567 (1994). D. Vollhardt and P. Wölfe, The Superfluid Phases of Helium 3, (Taylor and Francis, London, 1990). R. D. Pisarski and D. H. Rischke, Phys. Rev. Lett. 83, 37 (1999). T. Schäfer and F. Wilczek, Phys. Rev. D60, 114033 (1999). R. D. Pisarski and D. H. Rischke, Phys. Rev. D61, 051501 (2000). D. K. Hong, V. A. Miransky, I. A. Shovkovy and L. C. R. Wiejewardhana, Phys. Rev. D61, 056001 (2000). W. E. Brown, J. T. Liu and H. Ren, hep-ph/9908248. S. Hsu and M. Schwetz, hep-ph/9908310. D. V. Deryagin, D. Yu. Grigorev and V. A. Rubakov, Int. J. Mod. Phys. A7, 659 (1992). E. Shuster and D. T. Son, hep-ph/9905448. B. A. Freedman and L. D. McLerran, Phys. Rev. D16, 1147 (1977). V. A. Miransky, I. A. Shovkovy and L. C. R. Wiejewardhana, Phys. Lett. B468, 270 (1999). R. Rapp, T. Schäfer, E. V. Shuryak and M. Velkovsky, hep-ph/9904353, to appear in Ann. Physics. A. Chodos, F. Cooper, W. Mao, H. Minakata and A. Singh, Phys. Rev. D61, 46011 (2000). M. Rho, E. Shuryak, A. Wirzba and I. Zahed, hep-ph/0001104. M. Rho, A. Wirzba and I. Zahed, Phys. Lett. B473, 126 (2000). C. Manuel and M. H. G. Tytgathepph/0001095. G. W. Carter and D. Diakonov, hep-ph/0001318; Nucl. Phys. A661, 625 (1999). D. K. Hong, M. Rho and I. Zahed, Phys. Lett. B468, 261 (1999). T. Schäfer, hep-ph/9909574. S. R. Beane, P. F. Bedaque and M. J. Savage, nucl-th/0004013. K. Rajagopal and E. Shuster hep-ph/0004074. D. H. Rischke, nucl-th/0003063. D. K. Hong, Phys. Lett. B 473 (2000) 118. D. K. Hong, hep-ph/9905523. D. K. Hong, T. Lee, D.P. Min, Phys. Lett. B477 (2000) 137.

[6] M. Harada, F. Sannino, J. Schechter and H. Weigel, Phys. Lett. B384, 5 (1996) and see references therein for nuclear matter at low density.

[7] F. Sannino and J. Schechter, Phys. Rev. D52, 96 (1995); M. Harada, F. Sannino and J. Schechter, Phys. Rev. D54, 1991 (1996); Phys. Rev. Lett. 78, 1603 (1997); D. Black, A. H. Fariborz, F. Sannino and J. Schechter, Phys. Rev. D58, 54012 (1998); D. Black, A. H. Fariborz and J. Schechter, Phys. Rev. D61, 074030 (2000).

[8] G. 't Hooft, in: Recent Developments in Gauge Theories, eds., G. 't Hooft (Plenum Press, New York, 1980).

[9] F. Sannino, hep-ph/0002277. To appear in Phys. Lett. B 
[10] R. Ball, Phys. Rept. 182, 1 (1989) and see references therein.

[11] T. Appelquist, Z. Duan and F. Sannino, hep-ph/0001043. To appear in Phys. Rev. D. See also related papers: T. Appelquist, A. Cohen and M. Schmaltz, Phys. Rev. D60, 045003 (1999). T. Appelquist, A. Cohen, M. Schmaltz and R. Shrock, Phys. Lett. B459 , 235 (1999).

[12] C. Vafa and E. Witten, Nucl. Phys. B234, 173 (1984).

[13] S. Coleman, J. Wess and B. Zumino, Phys. Rev. 177, 2239 (1969). C. G. Callan, S. Coleman, J. Wess and B. Zumino, Phys. Rev. 177, 2247 (1969).

[14] D. H. Rischke, nucl-th/0001040.

[15] D. T. Son and M. A. Stephanov, Phys. Rev. D61, 074012 (2000); D. T. Son and M. A. Stephanov, hep-ph/0004095

[16] S. Raby, S. Dimopoulos and L. Susskind, Nucl. Phys. B169, 373 (1980). S. Dimopoulos and L. Susskind, Nucl. Phys. B173, 208 (1980).

[17] V. A. Miransky, I. A. Shovkovy and L. C. R. Wijewardhana, hep-ph/0003327.

[18] S. Hsu, F. Sannino and J. Schechter, Phys. Lett. B427, 300 (1998).

[19] R. Casalbuoni and R. Gatto, Phys. Lett. B464, 11 (1999); Phys. Lett. B469, 213 (1999).

[20] J. Schechter and A. Subbaraman, Phys. Rev. D48, 332 (1993). See also R. Casalbuoni, A. Deandrea, N. Di Bartolomeo, R. Gatto, F. Feruglio and G. Nardulli, Phys. Lett. B292, 371 (1992). M. Harada, F. Sannino, J. Schechter and H. Weigel, Phys. Rev. D56, 4098 (1997) and references therein. A. F. Falk and M. Luke, Phys. Lett. B292, 119 (1992); A. F. Falk, Nucl. Phys. B378, 79 (1992). For a review see: R. Casalbuoni, A. Deandrea, N. Di Bartolomeo, R. Gatto, F. Feruglio and G. Nardulli, Phys. Rep. 281, 145 (1997).

[21] S. R. Beane, P. F. Bedaque and M. J. Savage, Phys. Lett. B483, 131 (2000). For other discussions see also T. Schäfer and F. Wilczek, Phys. Rev. D60, 074014 (1999). 